\title{
Just sleep apnoea?
}

\section{Patient Case}

A 17-yrold man presented to the clinic with his mother after crashing his car. He had fallen asleep at the wheel and been referred urgently by his general practitioner.

He had driven for $1 \mathrm{~h}$ in the late afternoon without a break, prior to crashing. He denied feeling tired prior to the event and denied suicidal ideation. There was no history of substance or alcohol misuse, or of food consumption at the time, and no serious injuries were sustained as a result of the accident.

On further questioning, he had been excessively sleepy since the age of 14 yrs. On several occasions, he had fallen asleep standing up, during class and during exams. He stated that he could fall asleep within seconds if not concentrating on a task. His naps lasted for a few minutes only and he always awakened refreshed. At lunchtime, he took a nap of 20 mins and again in the evening on a regular basis. Nocturnal sleep was not disrupted. He described marked hypnagogic hallucinations at least once a month, both visual and auditory but denied sleep paralysis and cataplexy. His Epworth Sleepiness Score (ESS) was 18/24 and his mother scored him at 17/24 (the ESS is a subjective questionnaire based measure of sleepiness with a maximum score of 24 [1]). He retired between 22:00 and 23:00 h every night and got out of bed at 08:30-11:00 h. However, being a student at university, he admitted to an irregular sleep-wake schedule on occasion. Specific questioning about snoring

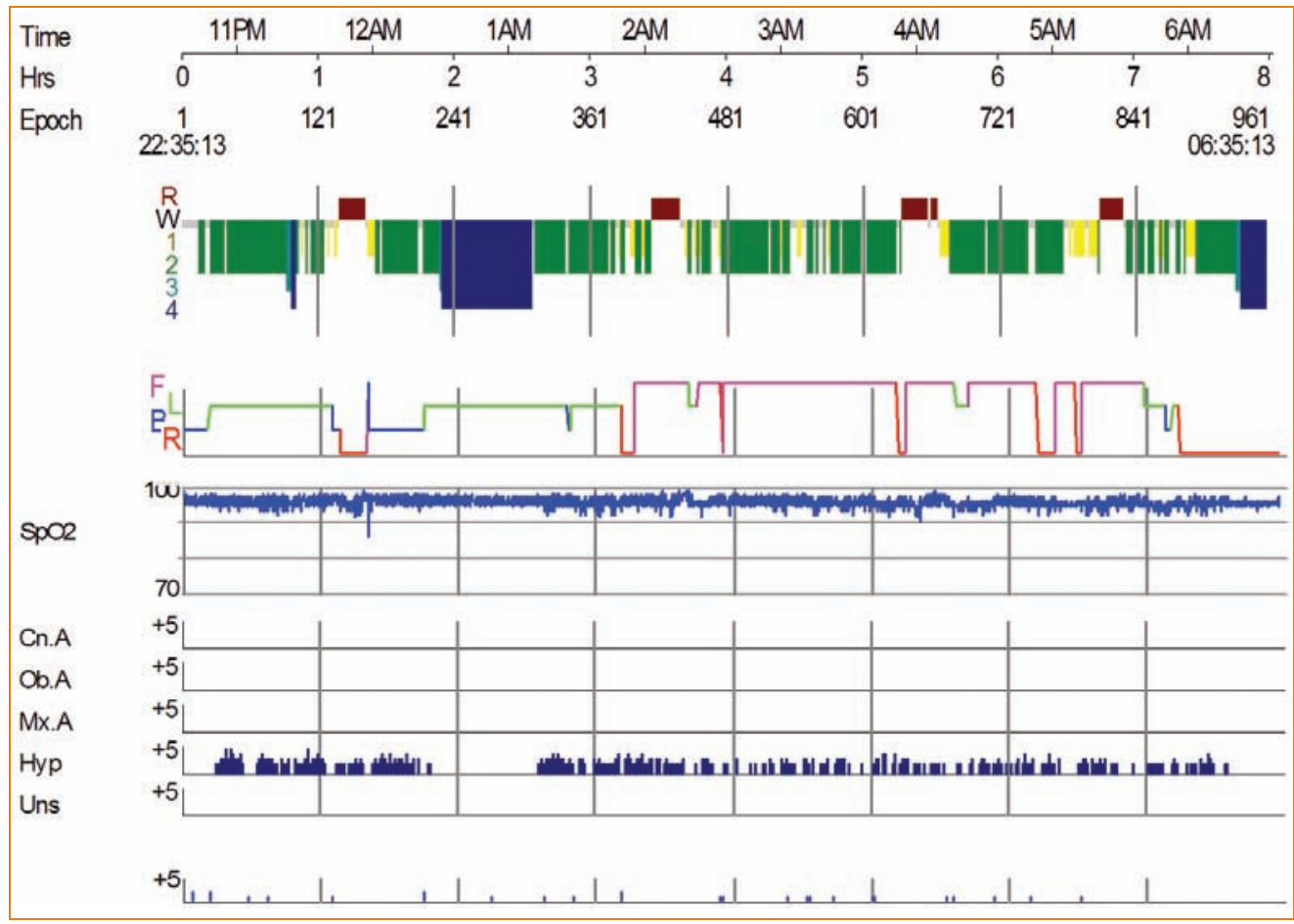

Figure 1

Polysomnography results.
R.L. Riha

Department of Sleep Medicine, Royal Infirmary of Edinburgh, Edinburgh, UK

\section{Correspondence}

Department of Sleep Medicine Royal Infirmary of Edinburgh EH16 4SA Edinburgh UK

rlriha@hotmail.com

\section{Competing interests}

None declared. 
revealed that he snored minimally but that it had been a feature since childhood. No apnoeas had ever been witnessed by co-sleepers or parents. He had a dry mouth in the morning and nocturia. There was no history of parasomnias and no family history of sleep disorders. His significant medical history revealed an allergy to peanuts which had previously lead to anaphylactic reactions. He was a nonsmoker and denied alcohol or recreational drug use. There was no significant family history of illness.

On examination, he was tall and slim, but not marfanoid. He had a height of $1.91 \mathrm{~m}$ and aweight of $85.7 \mathrm{~kg}$, giving him a BMI of $24 \mathrm{~kg} \cdot \mathrm{m}^{-2}$. His blood pressure was $120 / 70 \mathrm{mmHg}$ sitting. Cardiorespiratory and neurological examinations were normal. Examination of upper airway and nose revealed a narrow arched palate, the presence of allergic rhinitis, massive tonsillar hypertrophy, bilateral mucopurulent nasal discharge, a polypoidal middle turbinate and obstruction at the palatal level due to massive tonsillar hypertrophy.

The patient underwent overnight polysomnography in our sleep laboratory (fig. 1).

\section{Task 1}

Interpret the sleep study. 


\section{Answer 1}

Results of the sleep study showed a total sleep time of $6 \mathrm{~h}$ and 45 mins. He had a sleep efficiency of $84.7 \%$. His awake $\mathrm{Sp}_{1} \mathrm{O}_{2}$ was $96 \%$ on average and his nadir desaturation was $90 \%$. His apnoea/hypopnoea index (AHI) was recorded as 55.6 events $\cdot h^{-1}$ consistent with severe sleep-disordered breathing. There was no early onset REM sleep and no periodic limb movements, and no abnormal movements, consistent with a parasomnia, were seen.

\section{Treatment}

He was diagnosed as having severe obstructive sleep apnoea/hypopnoea syndrome (OSAHS) due to lymphoid tissue overgrowth and was commenced urgently on continuous positive pressure therapy (CPAP). Whilst on treatment, his ESS came down to $11 / 24$.

He was referred to the ENT surgeons who performed a tonsillectomy and removed the uvula which showed a benign squamous papiIloma. He stopped CPAP postoperatively. His ESS came down to 5/24 with resolution of all symptoms. He underwent repeat PSG (fig. 2).

\section{Task 2}

Interpret the post-surgical PSG.

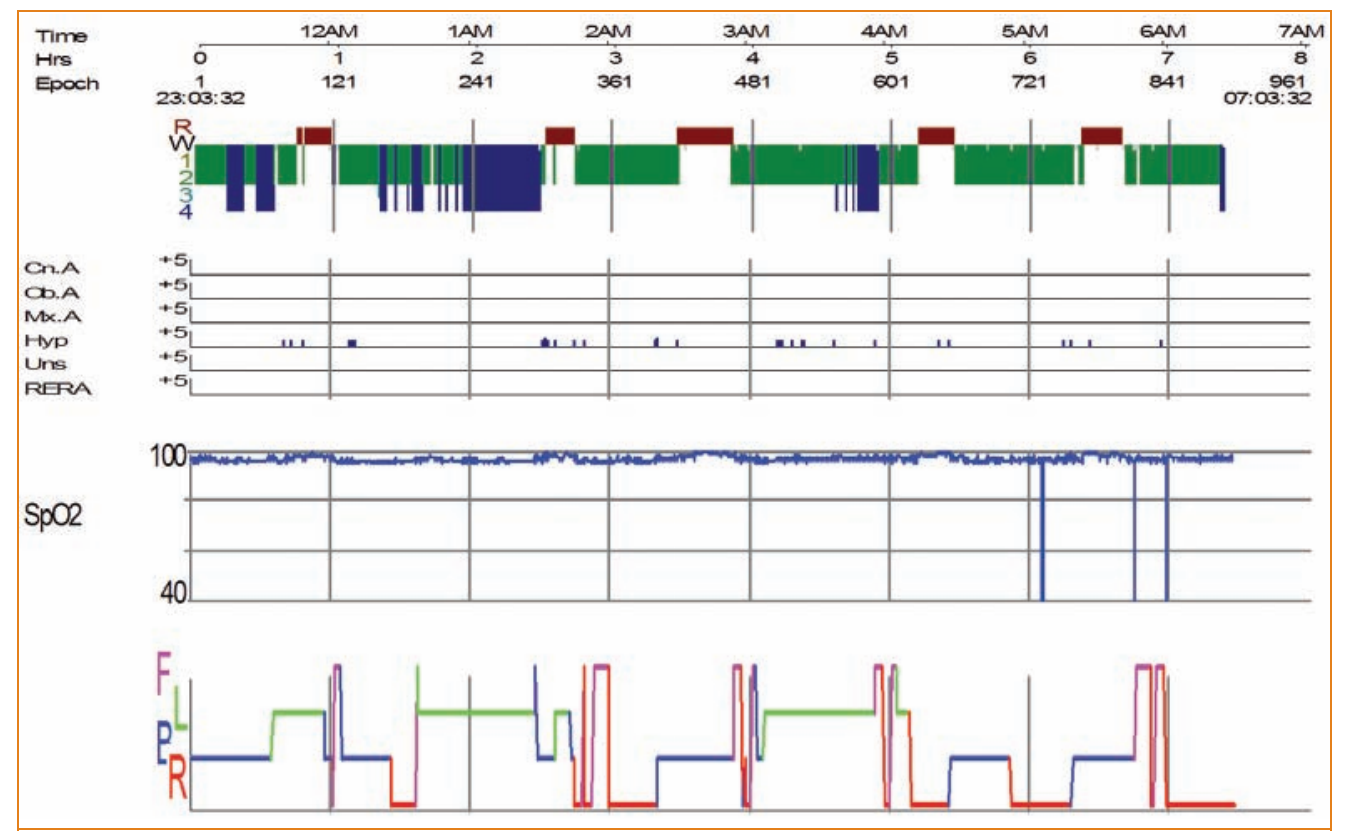

Figure 2

Post-surgical polysomnography. 


\section{Answer 2}

His post-surgical PSG revealed complete resolution of his sleep-disordered breathing (fig. 2).
The patient stopped using CPAP and now reported the following symptoms: in a state of intense emotion, he would feel weak at the knees on a weekly basis; he had episodes of feeling paralysed in bed; at times he would see

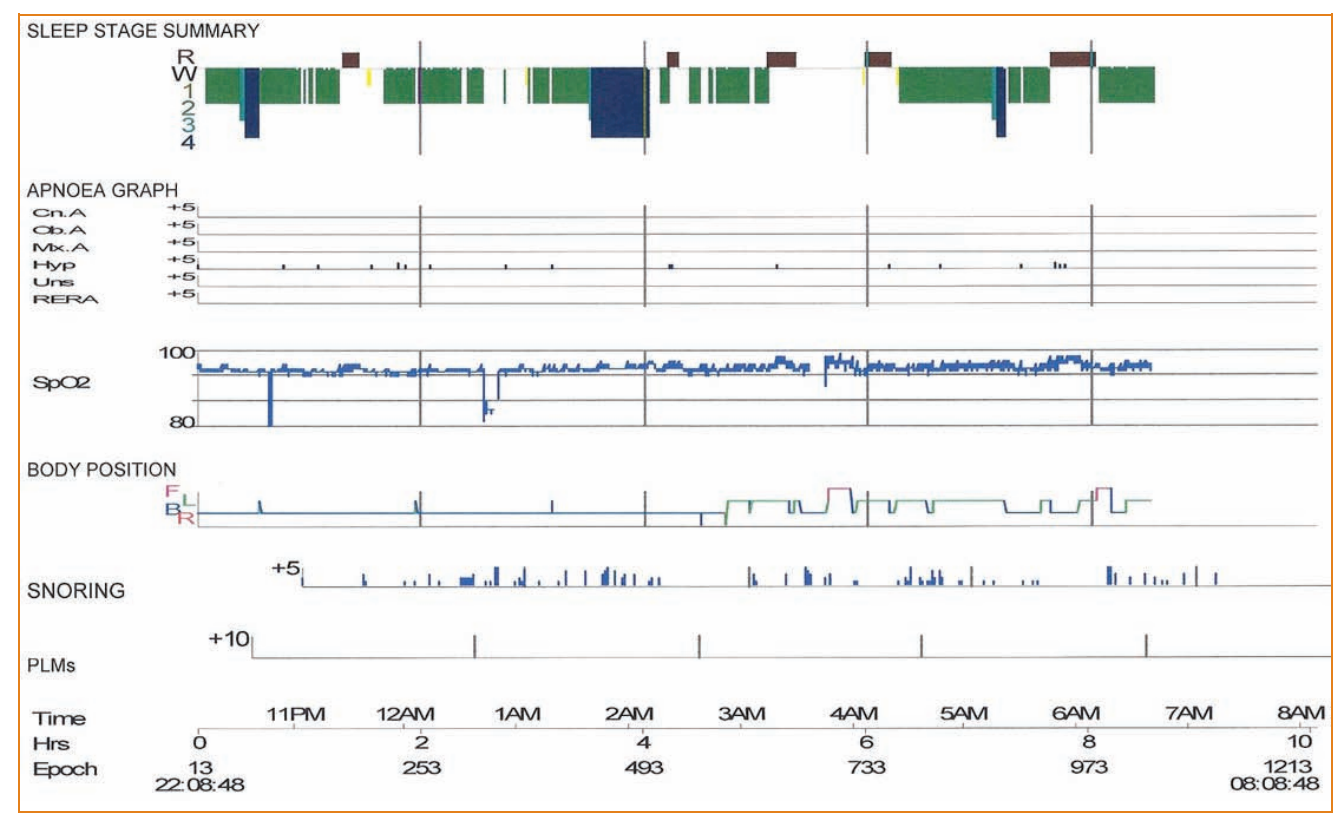

Figure 3

Repeat polysomnography

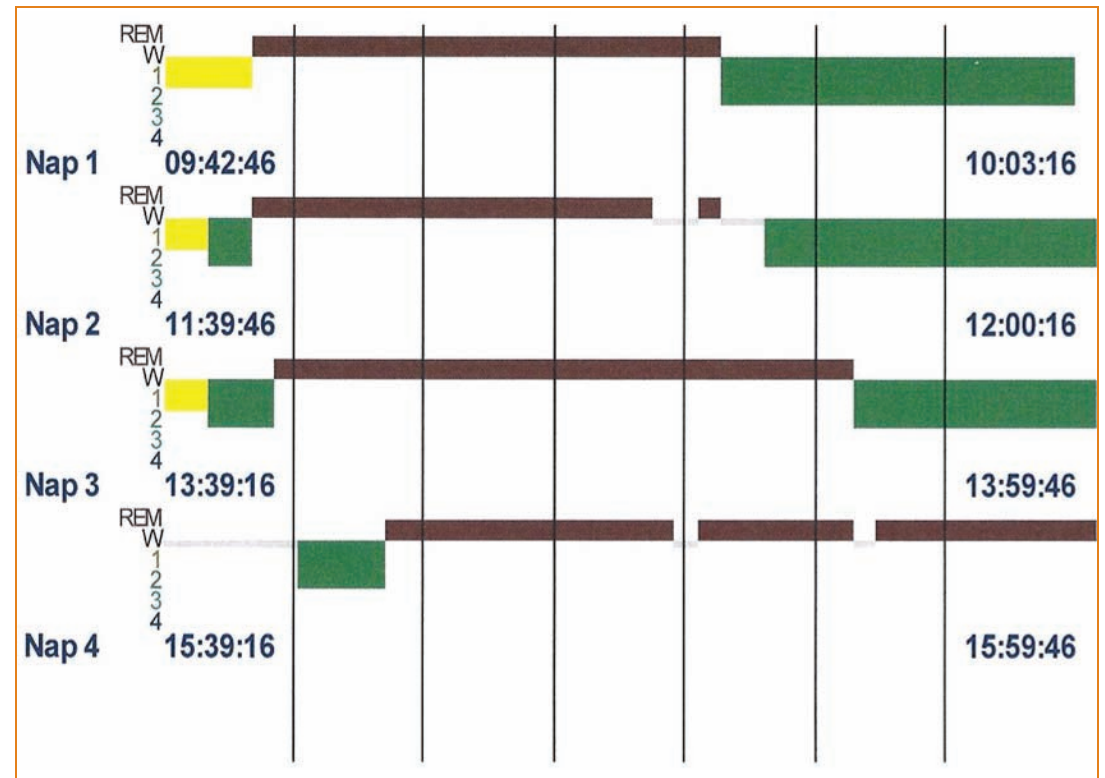

Figure 4

Multiple sleep latency test things coming out of the bookcase in the form of a hallucination.

However, he no longer felt sleepy at all but stated that he would fall asleep when travelling by bus. A sleep diary completed over a fortnight revealed a regular bedtime and awakening schedule with an average of 7-8 hours of sleep per night.

\section{Task 3}

What do the repeat PSG (fig. 3) and MSLT (fig. 4) show? 


\section{Answer 3}

The PSG showed a sleep efficiency of 69\% with no early onset REM. The patient's sleep latency was 5 minutes.

The MSLT performed the following day showed a mean sleep latency over four nap opportunities of 0.8 minutes and a mean REM latency over the same number of episodes of 2.1 mins.

Urine testing revealed no stimulant or sedative substances. The patient was diagnosed with narcolepsy.

He was commenced on $400 \mathrm{mg}$ Modafinil daily and $1 \mathrm{mg}$ of Clonazepam at night to good effect. Modafinil is a stimulant medication generally used as a firstline agent for treating the sleepiness associated with harcolepsy in the UK. Clonazepam is sometimes used in narcolepsy to treat nocturnal manifestations of the disease, such as sleep paralysis and hallucinations, including sleep disruption. He had minimal symptoms from his hypnogogic hallucinations, sleep paralysis and cataplexy. The patient continues to be followed up on a regular basis and is doing well.

\section{Discussion}

Excessive daytime somnolence (EDS) in adolescents presents a wide spectrum of differential diagnoses, both in terms of primary sleep disorders as well as those occurring secondary to medical and psychiatric conditions [2].

Probably the most widely encountered sleep problem in adolescence is that of insufficient sleep. Studies across populations have consistently shown that adolescents attending school sleep less during the week with extended sleep at weekends and during vacation [2]. Inade quate sleep not only leads to EDS but can have long-term effects on neurocognitive functioning, academic and work performance behaviour, mood and driving.

Despite insufficient sleep being a major problem in adolescence, it is essential to consider other causes of EDS and take a formal sleep history when faced with this problem. For instance, $7 \%$ of the adolescent population is said to suffer from the delayed sleep phase syndrome which may be exacerbated by environment and lifestyle [2]. The symptoms of narcolepsy often appear during adolescence and young adulthood, although the diagnosis may be delayed for many years due to poor recognition of the syndrome. Excessive caffeine intake and substance abuse may also contribute to sleep problems although the prevalence of this is currently unknown.

OSAHS affects $\sim 2-4 \%$ of the middleaged population. In adolescents, the true prevalence of OSAHS is unknown. There is a lack of studies in this area, in addition to the lack of consensus about minimum diagnostic criteria.

In younger children, an $\mathrm{AHI}$ of $\geqslant 1$ event $\cdot h^{-1}$ is considered diagnostic of sleep apnoea; this threshold may not be appropriate in older children and adolescents. One study has shown that an $\mathrm{AHI} \leqslant 3.6$ events $\cdot h^{-1}$ is normal in young boys [3].

The more common causes of OSAHS in the teenage group are likely to reflect that of both children and adults. In pre-pubertal children, the commonest cause of OSAHS is adenotonsillar hypertrophy, whereas obesity appears to account for the majority of OSAHS in adults [4]. However, there are a large number of other factors that contribute to the expression of sleep apnoea in these populations, including subtle craniofacial changes, possible problems with neuromuscular control of the pharynx, hormonal status and the impact of acquired conditions, environment and lifestyle.

Although surgery per se is not routinely advocated in the treatment of OSAHS [5], in this case, it effected a cure. If sleep apnoea is secondary to lymphoid tissue hyperplasia, current guidelines advocate surgery [6]. However, the evidence on which this is based is limited and at present, there is only one randomised controlled trial in children which examined radiofrequency ablation of adenotonsillectomy (AT) tissue versus surgical removal [7]. There are no such trials in adults and there have been no trials in either population comparing CPAP versus AT [5]. AT is considered curative in children. However, a recent meta-analysis has shown that AT alone has a success rate of $50 \%$ in obese children [5]. Severe OSAHS pre-operatively and persistent postoperative snoring are risk factors for persistent OSAHS $[4,5]$. The high cure rate quoted has recently been challenged and the need for additional treatments that complement AT proposed [4, 9]. Therefore, follow-up is recommended.

Sleep apnoea has also been found to have a significant association with narcolepsy and, if identified, may delay the diagnosis of narcolepsy by several years [10]. Diagnostic delay for narcolepsy of itself can be anything from 1 to 61 yrs with a median figure found in a large UK 
survey to be 10.5 years [11]. The initial absence of cataplexy may be the most important factor contributing to this delay [11]. However, most narcoleptic patients experience the initial symptoms of sleepiness prior to the age of 20 yrs $[12,13]$. Interestingly, treatment with CPAP does not usually improve EDS in patients with narcolepsy and sleep apnoea, but this can vary on an individual basis [10]. In this case, treatment of the sleep-disordered breathing led to a significant resolution of sleepiness and it is possible that the other symptoms of narcolepsy became more manifest after tonsillectomy and with increasing maturity of the patient.

The investigation and management of EDS in adolescence is not always straightforward and primary sleep disorders can go undetected for many years with potentially lethal consequences. Every patient who presents with EDS should have a structured sleep and medical interview with investigation and treatment directed at reversible causes. Much more work also needs to be done in the area of adolescent sleep medicine.

\section{References}

1. Johns MW. Reliability and factor analysis of the Epworth Sleepiness Scale. Sleep 1992; 15: 376-381.

2. Millman RP, et al. Excessive Sleepiness in Adolescents and Young Adults: Causes, Consequences, and Treatment Strategies. Pediatrics 2005; 11: 1774-1786.

3. Acebo C, Millman RP, Rosenberg C, et al. Sleep, breathing, and cephalometrics in older children and young adults. Part I-normative values. Chest 1996; 109: 664-672.

4. Praud JP, Dorion D. Obstructive sleep disordered breathing in children: beyond adenotonsillectomy. Pediatr Pulmonol 2008; 43: 837-843.

5. Sundaram S, Bridgman SA, Lim J, et al. Surgery for obstructive sleep apnoea. Cochrane Database Syst Rev 2005; 4: CD001004.

6. Won $\mathrm{CH}$, Li KK, Guilleminault C. Surgical treatment of obstructive sleep apnea: upper airway and maxillomandibular surgery. Proc Am Thorac Soc 2008; 5: 193-199.

7. Kuhle S, Urschitz MS, Eitner S, et al. Interventions for obstructive sleep apnea in children: a systematic review. Sleep Med Rev 2009; 13: 123-131.

8. Lim JC, McKean MC. Adenotonsillectomy for obstructive sleep apnoea in children. Cochrane Database Syst Rev 2009; 2: CD003136.

9. Verse T, Kroker BA, Pirsig W, et al. Tonsillectomy as a treatment of obstructive sleep apnea in adults with tonsillar hypertrophy. Laryngoscope 2000; 110: 1556-1559.

10. Sansa G, Iranzo A, Santamaria J. Obstructive sleep apnea in narcolepsy. Sleep Med 2010; 11: 93-95.

11. Morrish E, King MA, Smith IE, et al. Factors associated with a delay in the diagnosis of narcolepsy. Sleep Med 2004; 5: $37-41$.

12. Ohayon MM, Ferini-Strambi L, Plazzi G, et al. How age influences the expression of narcolepsy. J Psychosom Res 2005; 59: 399-405.

13. Nevsimalova S. Narcolepsy in childhood. Sleep Med Rev 2009; 13: 169-180. 